\title{
Nurturing context: TRACE, the arts, medical practice, and health literacy
}

\author{
John David Ike ${ }^{\mathrm{a}, *}$, Rachael Postlethwait ${ }^{\mathrm{b}}$ and Ruth Parker ${ }^{\mathrm{c}}$ \\ ${ }^{a}$ Duke University School of Medicine, Durham, USA \\ ${ }^{\mathrm{b}} \mathrm{MD}, \mathrm{UCF} / \mathrm{HCA}$ Consortium, Gainesville, USA \\ ${ }^{\mathrm{c}} \mathrm{MD}, \mathrm{MACP}$, Emory University School of Medicine, USA
}

\begin{abstract}
This article builds upon prior research on the interconnectedness of context to content as it relates to health and health literacy. The authors focus on the use of the arts as a novel way to engage with and to promote health and health communication. Most published efforts exploring the humanities and health emphasize how healthcare practitioners can enhance their clinical skills, promote wellness, and prevent burnout through engagement with the arts. The current article adds how the arts inform us about health, and more broadly, the lessons to be learned from appreciating multidimensional contexts. The authors underscore the role of the arts to address context and introduce how the arts impact health literacy. The authors provide an overview of TRACE, a unique pedagogical program that explores both the content and context communicated via the arts, with a focus on lessons for medicine and health, including health literacy. Special attention is given to the use of information technology services and its role in humanities and arts-based education. The article suggests enhanced provider apperception via arts exposure has implications to improve clinical practice and health literacy.
\end{abstract}

Keywords: Health literacy, the arts, context, content, information technology, internet, social media

\section{Introduction}

This article introduces a pedagogical program developed almost two decades ago by one of the authors [1]. The program, offered through Emory University's The Renaissance Academy at the Center for Ethics (TRACE), is a curricular immersion that encourages learners to glean lessons related to what the arts communicate as well as how arts advance the role - and understanding - of context and individual apperception. The authors participated as students and faculty in TRACE, which helps participants engage with the content communicated about health through the arts [1]. The latter experience develops an appreciation of the unique role the arts play in defining and utilizing context to communicate about health.

In this article the arts are operationally defined as fine or classical art without bias against popular culture that use drawing, painting, photography, sculpture, music, dance, theater, film, video, print and broadcast media as communication mediums. Apperception is operationally defined as one's ability to monitor the external world and simultaneously reconsider one's observations, knowledge, understanding of history, interpretation of cultural and professional paradigms, ideological framing, and social

\footnotetext{
*Corresponding author: John David Ike, MD, Internal Medicine Resident Physician, Duke University Health System, 2301 Erwin Road, Durham, NC 27701, USA. E-mail: john.david.ike@duke.edu.
} 
mores; understanding of qualitative and quantitative evidence, beliefs (or internalized truths), feelings, impressions, emotions, preferences, hunches, expectations, notions, and opinions [2]. Apperception (or the expansion of one's apperceptive mass or perceptual lens) is seen as an elastic skill integral to human development, which can be nurtured via formal education and informal learning [2].

As part of TRACE, medical students and undergraduate students (accompanied by practicing physicians and professionals from law, public health, and industry) engage in an intensive cultural immersion in Italy. The course explores themes of professionalism, compassion, and health through in-depth analysis of Italian culture, medical history, and the arts. Site specific visits and reflective writing foster an understanding of health-related context, and multidisciplinary discussions highlight the role of the arts in engaging context to communicate messages relevant to health and medicine. Engagement with the arts through internet based resources and multimedia allow participants to continue scholarship and assessment following on site visits.

Prior to departure, students and participants are introduced to visual literacy using an online Visual Thinking Strategies (VTS)-inspired curriculum, which provides a well-established method for interacting with visual content [3]. There are three central questions to answer when employing this method: What is going on in this picture? What am I seeing that is making me say that? What more can I find $[3,4]$ ? To articulate their experiences, students are asked to write a journal using a "See, Think, Wonder" framework, which echoes VTS with the following questions: What do you see? What do you think about what you see? What do you wonder? [4]. These journals, while analog, are frequently translated into digital forms for future sharing, collaboration, and reflection. Students also are exposed to the Toledo Museum of Art's 'Art of Seeing Art' curriculum, an online video series and methodology which builds upon the basic framework of VTS, but adds a vocabulary and analytical basis to deconstruct an image [5].

The authors suggest the TRACE program may be a pedagogical model to engage healthcare professionals (at all levels of training) with the arts to better relate to and ultimately communicate with patients and multidisciplinary teams in an increasingly multicultural and technologically complex health system. The authors additionally suggest the post-exposure expansion of physicians' apperceptive capabilities is consistent with at least two of health literacy's goals: (a) to generate interventions that are tangible, relevant, and patient-centered and (b) foster more thoughtful, interactive, and culturally competent patientcentered care [6].

The article provides an introduction to the prior literature about arts and health literacy, reviews selected literature about the impact of arts interventions in medicine, and notes a few studies where arts exposure is associated with improved participant understanding of specific diseases. A description of the pedagogical approaches within the TRACE program and a conclusion are provided.

\section{Prior literature about the arts and health literacy}

Several prior articles about the arts and health literacy provide a foundation for TRACE's pedagogical and professional training efforts as well as this article. In one article, Ike, Parker, and Logan emphasized the connection of context to communication and the extrapolation from understanding art to impacting health and healthcare experiences and improving health literacy [2,7].

In a second article, Parker and Ike introduce visual literacy as an underutilized component of health literacy [7]. Parker and Ike suggest the arts reflect shared human experiences and values, and such values infuse the understanding of content [7]. Developing visual literacy through purposeful implementation of validated techniques, including VTS and other related curricula, many of which are available online, 
enables healthcare practitioners to improve their ability to engage the visual world and its complexities, and by association fosters a deeper apperceptive appreciation for the contexts that are foundational to cross-cultural communications [7]. Parker and Ike suggest medicine and health are inextricably linked to culture [7]. In turn, patients and communities are more likely to engage in activities that promote wellbeing (medication compliance, exercise, community engagement, education, etc.) if content is articulated within an appropriate context [7].

In this subsequent commentary, Parker and Ike argue the arts are underutilized in understanding and communicating the contextual dimensions of perception [7]. Parker and Ike suggest efforts to preserve, revere, and study the arts reflect an appreciation for culture and human value systems [7]. Using the Smoketown Lifeline Project as an example, Parker and Ike argue Andrew Cozzens' sculptural installation within an impoverished community center manifests health inequity and creates a platform to broaden discussions about health disparities [7].

Other research about the connections between content, context, the arts, and health has focused on specific health outcomes. For example, Schillinger recently suggested the effectiveness of a social and culturally cognizant mass communication campaign about the prevention of type 2 diabetes [8]. In a video intervention, entitled The Bigger Picture, young minority poets produced spoken word public service announcements (PSAs) for at-risk youth regarding the prevention of type 2 diabetes. The project's intent was to reframe a spike in obesity-related type 2 diabetes among California youth within a novel socio-environmental context targeted at young minority populations. The rap-music grounded poets focused on aspects of diabetes prevention and communicated through the perspective of impacted at-risk peer adolescents.

The campaign's assessment suggests full recognition of the nine PSAs' intended public health messages ranged from $22.2 \%$ to $70 \%$ among intended audiences. On average, $43 \%$ of respondents demonstrated a comprehensive understanding of the intended message about type 2 diabetes' prevention. The use of video arts and poetry suggested: "the ability of [a] spoken word medium to make these challenges [related to diabetes education] visible and motivate action." Schillinger et al. suggest the arts have a unique ability to "change behavioral norms and build capacity to confront the social, economic and structural factors that influence behaviors" [8]. In essence, the arts offer a different means to engage content and appreciate cultural context.

Some North American medical schools have also implemented arts-based programs in an effort to train compassionate and cognizant health care workers, who understand and can navigate the mosaic of cultures providers are licensed to serve. Arno Kumagai, a Professor of Medicine and Vice Chair for Education at the University of Toronoto has done extensive work in the medical humanities. In one of his short pieces, “"Making Strange': A Role for the Humanities in Medical Education," he describes five distinct ways in which engagement with the arts improves the training of physicians and improves patient care $[9,10]$. First, Kumagai suggests the arts serve as a form of disruption $[9,10]$. The arts complicate and obscure commonly held beliefs and practices by presenting them in new and different lights $[9,10]$. As highlighted in an analogous piece, the arts 'makes strange' and "distort our perceptions of common objects, relationships, ideas, identities, or beliefs to force us to look at them anew" $[9,10]$. The history of art is populated with examples, such as Dadism, Abstract Expressionism, and Surrealism, that attempted to redefine the perception of visual reality in the first half of the 20th century.

Kumagai adds arts and humanities teach us to pause. For clinicians, arts exposure interrupts the automaticity of much of clinical practice and requires a viewer to wrestle with ambiguity and uncertainty. Since evidence-based uncertainty is ubiquitous in clinical practice, the arts function as a tool to enhance providers' capacities to reconsider how ambiguity is perceived and communicated. This additionally 
improves how medicine's uncertainty is communicated, which potentially enhances patient/provider interactive health literacy.

Kumagai also explains engagement with the arts tasks providers to imagine the environment their patients inhabit $[9,10]$. Kumagai suggests "[the arts] help us to peel back the different layers that compose up ideas, institutions, conditions, or practices to understand them through the lens of history, ideology, and power" $[9,10]$.

Kumagai adds the arts offer a means for physicians to exercise one's moral imagination, or to ponder the experience of others while paying particular attention to their innate humanity [11]. Kumagai emphasizes the role of literature to transport a reader into the mind of characters and how such acts develop a compassion and empathy for the lived experiences of others. Finally, Kumagai notes engagement with the humanities and arts enable a broader understanding of the human experience and by association "ultimately...prompt awareness of the space in which physicians care for human beings in their moments of greatest need and bear witness to fundamental changes in their patients and in themselves" [9].

In summary, the authors suggest arts exposure potentially advances a provider's apperceptive capabilities that aggregate many of the professional developmental dimensions which Kumagia cites $[9,10]$. Learnings are enhanced by the richness of online repositories of art work and easy access to scholarly interactive discussions about context, content, and analyses of meaning.

\section{The TRACE 2018 colloquium: exploring evidence, truth, and wisdom communicated through the arts}

The 2018 TRACE colloquium involved medical students, undergraduates, and professionals from the fields of medicine, ethics, law, and the humanities who journeyed together to several Italian cities including Rome, Pisa, Florence, and Siena. The nine-day colloquium uses arts immersion to help future and current physicians appreciate and understand cross-cultural biopsychosocial, economic, and cultural contexts.

Site visits and city explorations were intentionally paired with selected written works and visual art to integrate three themes: (1) communication and compassion; (2) death, dying, suffering; and (3) beauty, balance, harmony. A prior qualitative analysis by course faculty described the origins of these central themes using modified crowd-sourcing [1].

The authors view TRACE as a unique exercise to expand health professionals' apperception as well as inculcate an enduring understanding of patient and provider health literacy. The decision to focus on the contextual nature of evidence, truth, and wisdom during site visits (or apply enduring questions in diverse settings) additionally helps participants turn the consideration of foundational constructs into a habitual skill. Engagement with the arts and humanities through online resources including Google Arts and Culture among other archives enabled students and participants to continually revisit evidence prior to, during, and after the course.

The following subsections describe how paintings and architecture can be utilized to challenge a health professional's apperception of three enduring clinical questions: what is evidence, what is the truth, and how does one derive wisdom when evidence and truth are equivocal.

\subsection{Art and consideration of what is evidence?}

The TRACE Colloquium first turns to the arts as a medium to explore the strengths and limits of evidence, or ask what is evidence? 


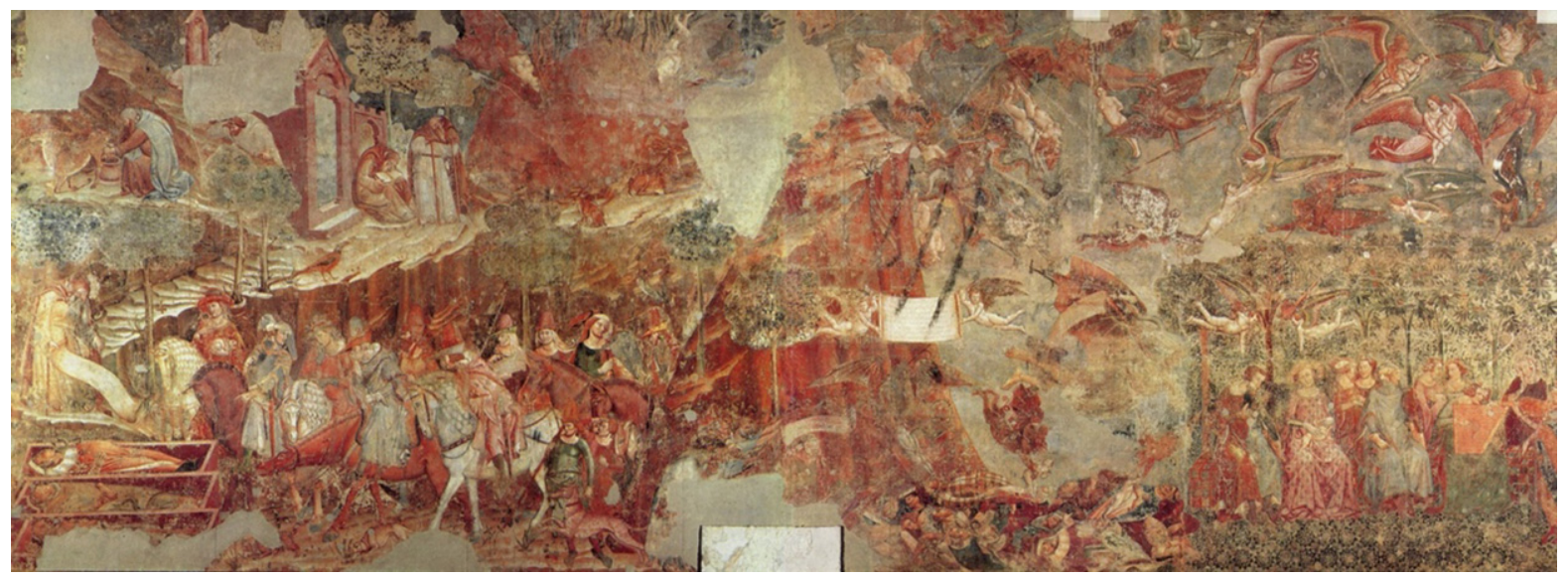

Fig. 1. Buonamico Buffalmacco, The Triumph of Death, 1336, Pisa, Italy. [Public Domain]

The arts featured during TRACE frequently focus on the Black Death, a plague that ravaged Italy from 1347 to 1351 . The visual evidence of the plague is especially well-captured in the Trionfo della Morte (The Triumph of Death) fresco in a remarkable cemetery in Pisa that dates from this period (Fig. 1).

Painted by Buonamico Buffalmacco in the mid-1300s shortly after the arrival of the plague in Pisa, the fresco depicts a complex scene. On the left side of the composition, those bedraggled by illness beg for death's mercy while a personification of the Grim Reaper turns instead to bring death to courtly ladies and gentlemen depicted on the right side of the composition. In essence, the fresco communicates the universality of death and its disregard for established cultural castes. At the time of horrific death, dying, and suffering, this painting communicated to the predominately illiterate citizens of Pisa a universality; the plague comes for all. It is instructive about a belief system and offers layers of complexity surrounding broader themes of death.

Despite its creation nearly seven centuries ago, the fresco remains instructive and offers its observers an opportunity to reflect. For example, TRACE participants were asked to discuss death and loss in their own professions and personal lives. Participants were encouraged to journal about their experiences in both analog and digital formats to enable continued reflection and integration of experiences at later dates. Sharing of content through social media outlets-Instagram, Twitter, and Facebook—enabled a continual and evolving conversation with the content. It also afforded means to engage friends and colleagues not present in Italy.

Given its setting within a monumental cemetery next to the well-known Pisa Cathedral complex (and its Leaning Tower), TRACE participants probed additional existential questions such as: What does it mean to honor the dead in times of great loss? How did the population respond? How would I have responded, and why would I respond that way? What motivates a city to construct such a beautiful and monumental structure for burial of their dead? What are the lessons of the Triumph of Death fresco that transcend time? Is the fresco evidence of the sense of loss, suffering, and the scale of population decline that occurred in Pisa during the Black Death? Other than newer mass mediums and data, what are some key differences between contemporary and medieval interpersonal and mass communication about death and catastrophes? Are contemporary efforts to inform impacted populations about public health emergencies occasionally culturally divisive and dysfunctional? Are the dysfunctional efforts a byproduct of health disparities and limited population health literacy? How has the internet increased our awareness 
of catastrophic health events and disparities globally? Does social media change the way we perceive, engage with, and understand humanitarian crises and health? Does it alter how we interpret evidence?

As Pisa provided an opportunity to consider evidence about death, dying, and suffering, a hospital in Siena encouraged participants to consider themes regarding evidence, human compassion, and public communication. Santa Maria della Scala, which is Europe's oldest hospital, is located across the piazza from Siena's Grand Cathedral [12]. Built initially to care for those traveling the Via Francigena, a pilgrimage route linking Rome to Gran San Bernado, this ospedale later became Siena's and Europe's original healthcare institution.

As the hospital grew and acquired significant landholdings to support its mission to the poor and sick, artist Domenico di Bartolo and his workshop were charged with decorating the Pellegrinaio (central entrance hall) which served as a vestibule to welcome visitors, volunteers, and the city's needy populations (Fig. 2).

Painted in the early 15th century shortly after the Black Death wiped out much of the Italian (and European) population, the fresco cycle communicates the function of the hospital and its role in caring for pilgrims, orphans, the poor, and the sick. The diverse panels depict the founding of the hospital, the care for orphans by wet nurses, the feeding of pilgrims at a communal dining table, and the intimate care for the dying and sick.

The caring, provision, receiving, and other messages about healing are expertly conveyed. The content is fundamentally linked to Siena's 15 th century as well as its enduring cultural values and belief systems about life, death, health, and public welfare. The contemporary efforts to preserve the fresco cycles through charitable donations and the building's conversion into a museum suggest the paintings' capacity to communicate through time and space. Moreover, the decision to digitally preserve the work through photographs and curated online modalities highlight the universality of the themes and the role for continued global engagement.

The images portray themes of hospitality and compassion and serve as one of the earliest known efforts to suggest the centrality of individual and public health to social welfare, the needed capacity for health care providers to utilize evidence to benefit individuals and society, and the importance of providers and citizens to care about the welfare of others. In short, the frescos provide qualitative evidence regarding what values should underlie health care and the appropriate role for society to care for the sick, disabled, and underprivileged.

The TRACE participants who engaged the Siena fresco cycles were asked to ponder an array of questions related to their own respective institutions, such as: outside of mission statements and websites, are there additional physical manifestations of your hospital's value system? If so, is this clear to providers, patients, or both? Why does communicating institutional values provide an opportunity to elevate health literacy as well as demonstrate institutional goodwill? What can one of the world's first public hospitals teach us about the principles of hospitality and healing? Does your hospital use social media? If so, does it use art to communicate themes of health and healing?

Albeit challenging to answer, the Siena frescos provided a means to discuss some of the current sociocultural challenges for health care institutions as well as their role in improving medical practice and contributing to their surrounding communities. The Siena frescos provided an opportunity to discuss the need for clinical institutions to integrate health literacy principles within routine practice and in outreach to patients, caregivers, and communities. The frescos also provided a means for participants to discuss the role of art in community and individual healing and the importance of provider humility and socio-cultural compassion. 


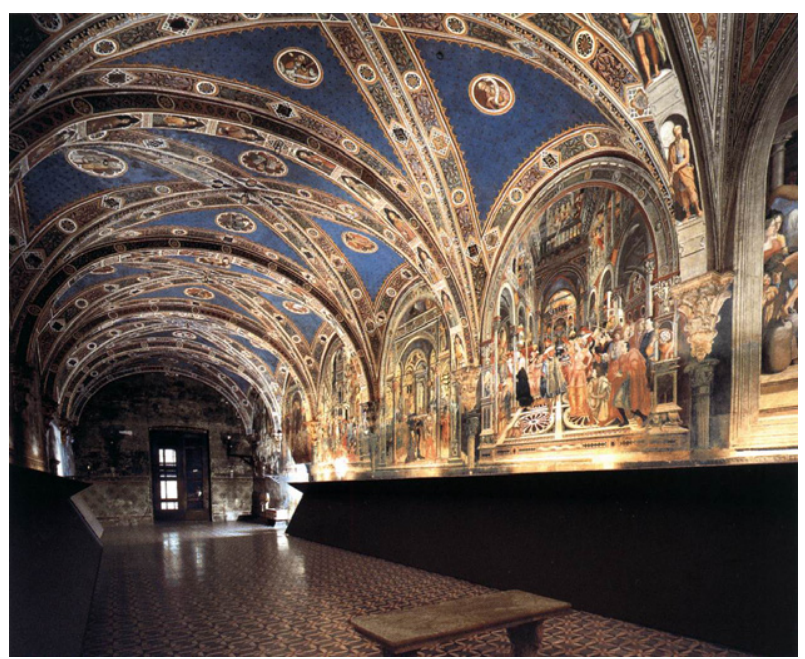

Fig. 2. Domenico di Bartolo and others, Pellegrinaio of Santa Maria Della Scala, Siena, Italy. Early 14th Century. Museum. (Shared with Permission)

In addition to exploring the role of hospitals and health systems in medieval Italy, TRACE explored other civic institutions including orphanages. Humanism flourished in fourteenth and fifteenth century Florence and the latter cultural movement fostered a new interest in civic and social responsibilities that spawned the start of public orphanages (among other advances).

The Ospedale degli Innocenti (Hospital of the Innocents), an orphanage in Florence, illustrates civic responsibilities, the plague's impact, and a response to social stigma and dysfunction. Following the Black Death, Florence's orphans increased significantly. In a 1419 effort to meet a growing demand, the civic government and the Silk Guild contracted Brunelleschi, an architect renowned for his work on Florence's Duomo Cathedral, to design the Spedale.

Utilizing a restrained design that blended both the Romanesque and Gothic architectural vocabularies, Brunelleschi created a system of repeating arches that evokes harmony and balance - the height of each arch is directly equal to the distance between columns. Between each arch, spandrels were decorated with tondi that were later filled with porcelain sculptures of swaddled infants by Andrea della Robbia. Underneath the arched portico was a 'wheel' that enabled families unable to care for their infants to anonymously deposit their children into the care of the orphanage's staff.

At this site, participants were asked to discuss the architectural principle of 'form meets function' and the role of design in communicating the values and legacies of social institutions and their civic sponsors. The Florentian structure, through its architectural vocabulary and civic purpose, also suggests the importance of civic duties in times of community strife. The restrained use of inexpensive but elegant building materials reinforces frugality, but not at the cost of beauty and harmony. The building's preservation and conversion to a museum, which was completed in 2016, suggests the enduring values the structure has conveyed through seven centuries of Florence's history. This history is further reflected in digital archives and sources within the museum that enable visitors and guests to trace the lineage of individual orphans in the same way that Ellis Island visitors in the United States can track the immigration of their ancestors.

In terms of evidence, the orphanage additionally conveys a 15th century understanding of the need to counter family/personal stigma via a social commitment to care for the city's children. 


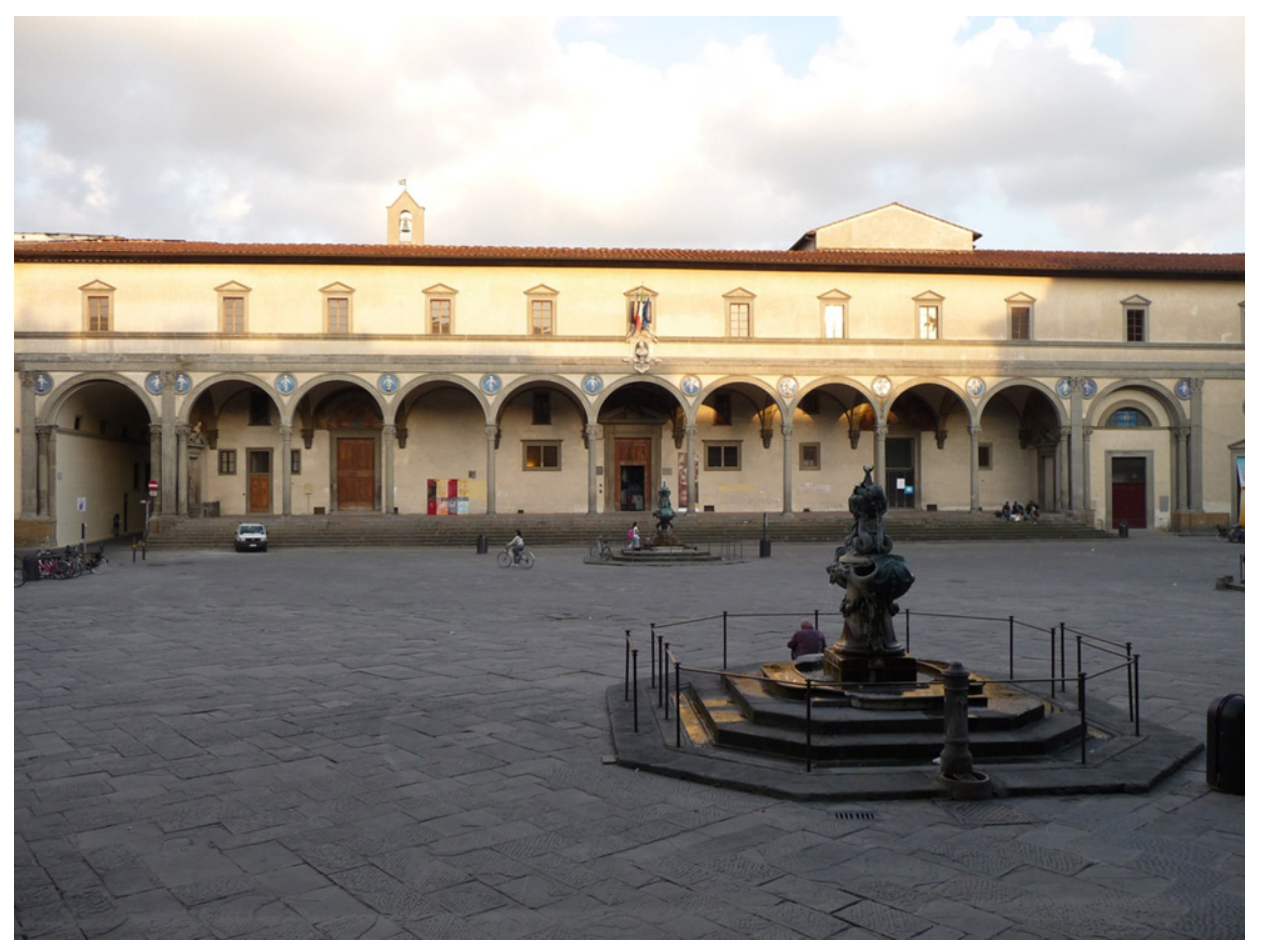

Fig. 3. Filippo Brunelleschi, Hospital of the Innocents, early 15th century, Florence Italy. (Credit: Warburg)

Using the site as inspiration, TRACE participants were asked to consider human experiences in times of catastrophe. What evidence does the orphanage structure suggest about medieval Italy's frailty during a time of great strife? Similarly, participants were asked to consider more recent, significant international epidemics such as the Spanish Flu of 1919 and the ongoing Ebola epidemic in West Africa. Participants also discussed Camus' The Plague, which describes the impact of the same disease in a fictional mid-20th century Algerian village.

Subsequent TRACE conversations included the scarcity and shortages of clinical essentials, such as vaccinations, tertiary medical care, and medications both today and in past centuries. Some of the other discussed issues included: How does the Florentine structure provide evidence about how people responded to the plague 700 years ago? How do we respond to contemporary catastrophes? What is a health providers' duty to humanity, and has this changed with time and circumstances? How should health care professionals attempt to overcome social stigma when there is cultural apathy or rejection of clinical evidence that adversely impacts individual and public health? To what extent should medical professionals be vigilant when popular efforts represent threats to public health, such as current antivaccination factions, or the prevalence of easily preventable diseases that are exacerbated by routine behaviors such as an imbalanced diet, smoking, lack of exercise, and substance abuse?

Utilizing the plague to explore enduring themes, Italy's architectural and artistic treasures serve as primary sources to explore the nature of qualitative, observational evidence and how this differs from the quantitative evidence that undergirds contemporary clinical practice. The art raises questions about the enduring aspects of historical evidence and learning from history. The experiences additionally raise questions about the roles and responsibilities of medical professionals and the importance of improving 
the public understanding of individual and public health, which are important dimensions of health literacy [13].

\subsection{Art and consideration of what is truth?}

The arts in Italy enabled consideration of the nature of enduring truths as well as the strengths and limits of contemporary clinical protocols derived from evidence-based data. The content and context provided by the aforementioned Pisa, Florence, and other examples raise consideration of diverse truths among professions and religions, as well as the need for a tolerance of ambiguity to assess and communicate multidimensional social and clinical challenges.

More specifically, TRACE participants were exposed to Plato's Allegory of the Cave from The Republic because of its exploration of truth's complexity and its link to context and apperception.

The hospital frescos in Siena and Pisa suggested the artists' understanding of the contrasts and paradoxes between religious and professional truths, as well as the enduring limits of the later to provide enlightened responses during public emergencies. For example, the Siena and Pisa paintings posit divine intervention was sufficient to explain the death and destruction that occurred during Italy's medieval plague, while also capturing the secular challenges health providers (and family members) faced during an overwhelming tragedy and civic emergency.

In other words, the paintings (and Camus' writing in The Plague) suggested how perceived truth's differing dimensions are impacted by religion as well as by contemporary cultural and professional assumptions and protocols.

For TRACE participants, the arts exposure also raised specific discussions about the differences in the determination of truth among diverse professions. TRACE visits included discussions of how evidence and fact are perceived differently among attorneys, journalists, scientists, medical professionals, and clergy, and how these sometimes contrast (or are consistent) with cultural beliefs. The discussion often triggered insights among participants about the importance of personal and professional information seeking, the need to occasionally challenge professional predispositions, and the need to challenge social norms. It demands a continual re-evaluation of primary resources both on site and through verified online databases of texts and artistic works that serve as a primary resource extender. As participants considered the intricacies of truth, questions emerged such as: How does evidence relate to and inform truth? Is truth eternal, or relevant to time and place? What is the price of being on the side of truth? How is truth related to health?

\subsection{Art and apperception: deriving wisdom when evidence and truth are equivocal}

The arts visited during TRACE also depicted liminality, or the challenging leap of faith health professionals are forced to make to derive contextual wisdom (the best available response) when clinical evidence and larger truths are equivocal, uncertain, ambiguous, or conflicting.

To illustrate this flow of decision making and to consider the nature of wisdom, TRACE participants engaged with art that represented a liminal space. A liminal space is "a threshold, a crossing, an in-between state that is neither here nor there" [14].

Liminality was operationalized by a 5th century BC sarcophagus now housed in an archaeological museum in Paestum, Italy. Intricately decorated with frescoes on its internal surfaces, the sarcophagus' inner lid faces the deceased so it cannot be viewed externally by onlookers. The frescos depict the image of a man suspended in flight between a diving pier and a body of water below. His journey is thought to represent the voyage from the world of the living to a spiritual home amongst the dead. 
However, the deceased is presented within the unknown space between life and death rather than either of their realities. The deceased is suspended in flight for eternity. The conundrum is a metaphor for liminal space and the unpredictability found in the limbo where contemporary professional and humane decisions often arise.

While the Paestum sarcophagus represents limbo, it also provides a space to find wisdom in spite of prevalent uncertainty. In addition, the Paestum sarcophagus metaphorically suggests: (a) the self-reflective space health professionals need to nurture so they are considerate during difficult decisions; and (b) the need to empower patients and caregivers to overcome the conundrum of equivocal health care decisions. The centrality of patient and caregiver empowerment often are presented as foundations to enhance health literacy and improve specific patient health outcomes $[15,16]$.

\subsection{The internet, social media, and multimedia resources. What can they teach us of evidence, truth, and wisdom?}

With the dawn of the internet on January 1, 1983, access to information has grown at an exponential pace. It has demanded, in addition to use of visual literacy, the development of digital literacy and media-specific apperception. Content from verified and unverified sources abounds and it is of increasing importance that professionals at all levels learn to engage with this material in thoughtful and critical ways. The introduction of social media in the 2000s introduced a secondary layer of complexity that has amassed millions of users and permits sharing of various content to diverse audiences. Throughout the TRACE course, both social media and engagement with primary resources through various art and humanities databases and repositories promoted continued interaction with content and by association an appreciation for context.

Participants were encouraged to use internet-based resources prior to departure, while traveling, and upon return to seek broader understanding of evidence, truth, and wisdom. The introduction to VTS and Toledo Museum of Art's 'The Art of Seeing Art' online curricula before venturing through Italy primed participants with the necessary vocabulary and skills to analyze and interpret works of art; to engage in conversations related to a medium outside many comfort zones. Exploration of this content in situ and thereafter through digital databases permitted continual development of visual literacy skills.

Throughout the journey, participants documented their experiences in photographs, journals, and brief posts both digital and analog. Many of these observations were shared via various social media outlets (Facebook, Instagram, Twitter). This enabled continued conversation and engagement among participants on site and with colleagues abroad.

We argue that these various internet based modalities offer access to content and promote a continual engagement with evidence and a broader appreciation of the global context in which health and healthcare practices take place. Art databases, in effect, serve as a primary source extender, constantly available to challenge beliefs, inform viewers, and foster culturally competent citizens and healthcare practitioners.

\section{Conclusion}

The authors suggest the TRACE program is a pedagogical model to engage healthcare professionals (at all levels of training) with the arts to better relate to and ultimately communicate with patients and multidisciplinary teams in an increasingly multicultural and technologically complex health system. 
The aggregate experiences during TRACE reflect all five of Kumagai's aforementioned goals to engage the meaningful professional development of physicians and other health care providers. TRACE provides an intervention that directly impacts many of apperception's multidimensional levels, which ultimately fosters a provider's professional growth and development.

Through an appreciation of culture and context, a physician's ability to communicate across time and cultural boundaries improves, becomes more sincere, and ultimately, more humane. The authors posit that dialogues based on mutual understandings of context enhance patient-centered care. As technology increasingly dominates the cultural landscape of the clinical practice of medicine in traditional healthcare settings, novel efforts become correspondingly exigent to maintain and enhance meaningful interpersonal communications.

As the arts build apperception, or the capacity to interconnect context to immediate content and immediate experience, TRACE provides a range of interventions that imbue contextualization. The authors suggest similar experiences with the arts can occur in many communities and through various online forums, as health professionals, educators, patients, and others engage appropriate catalysts.

Finally, the authors suggest TRACE fosters at least two of health literacy's primary goals: (a) to generate care that is tangible, relevant, and patient-centered and (b) to foster more thoughtful, interactive, culturally competent, patient-centered care. To advance health literacy, there is no substitute for a multidimensionally trained workforce who is able to engage and empower patients, caregivers, and surrounding community utilizing a variety of traditional and technologically innovative internet-based approaches.

To advance health literacy, both content and context deserve careful consideration. The arts represents an underappreciated opportunity to utilize context to enhance communication and promote the prevention of specific diseases, emotional and psychology health, and create a dialogue about public health challenges. With growing reliance on digital connectivity both within health systems and the community at large, attention to the arts within digital health and healthcare space appear ripe for exploration and represents an emerging frontier for continued patient and provider engagement.

\section{References}

[1] R.M. Parker, C.A. Labrecque, S.G. Candler, D. Newell-Amato, J. Messler, M. Wolf et al., Communicatin through the arts: lessons for medicine and public health, J Health Commun 18(2) (2013), 139-145. doi:10.1080/10810730.2013.763706.

[2] J.D. Ike, R.M. Parker and R.A. Logan, Health literary and the arts: exploring the intersection, Stud Health Technol Inform 240: (2017), 81-95.

[3] https://vtshome.org/about/. Retrieved April 22, 2019.

[4] J.M. Reilly, J. Ring and L. Duke, Visual thinking strategies: a new role for art in medical education, Fam Med 37(4) (2005), $250-252$.

[5] http://www.vislit.org/the-art-of-seeing-art/. Retrieved April 22, 2019.

[6] K. Sorensen, S. Van den Broucke, J. Fullam, G. Doyle, J. Pelikan, Z. Slonska et al., Health literacy and public health: a systematic review and integration of definitions and models, BMC Public Health 12(80) (2012). doi:10.1186/1471-245812-80.

[7] R. Parker and J.D. Ike, Health literacy and the arts: an intersection worth exploring, (2017). doi:10.31478/201707e.

[8] D. Schillinger, J. Tran and S. Fine, Do low income youth of color dee "the bigger picture" when discussing type 2 diabetes: a qualitative evaluation of a public health literacy campaign, International Journal of Environmental Research and Public Health 15(5) (2018). doi:10.3390/ijerph15050840.

[9] A.K. Kumagai, Beyond "Dr. feel-good": a role for the humanities in medical education, Acad Med. 92(12) (2017), 16591660. doi:10.1097/ACM.0000000000001957.

[10] A.K. Kumagai and D. Wear, "Making strange": a role for the humanities in medical education, Acad Med 89(7) (2014), 973-977. doi:10.1097/ACM.0000000000000269.

[11] http://www.firstthings.com/blogs/firstthoughts/2009/07/defining-moral-imagination. Retrieved February 11, 2017. 
[12] J.H. Baron, The Hospital of Santa Maria della Scala, Siena, 1090-1990, BMJ 301(6766) (1990), 1449-1451.

[13] R.A. Logan, Health literacy research, in: Meeting Health Information needs Outside of Healthcare: Opportunities and Challenges, C. Arnott-Smith and A. Keselman (eds), Chandos, Waltham, MA, 2015, pp. 19-38.

[14] C.A. Labrecque, In over one's head: the tomb of the diver and the 'Technologization' of dying, Acad Med 89(7) (2014). doi:10.1097/01.ACM.0000451521.36698.69.

[15] M.A. Abrams, P. Klass and B.P. Dreyer, Health literacy and children: recommendations for action, Pediatrics 124(Suppl 3) (2009), S327-S331. doi:10.1542/peds.2009-1162I.

[16] D.A. DeWalt and A. Hink, Health literacy and child health outcomes: a systematic review of the literature, Pediatrics 124(Suppl 3) (2009), S265-S274. doi:10.1542/peds.2009-1162B. 